REIMPRESIONES Rev Chil Salud Pública 2013; Vol 17 (3): 290-297

\section{PAPEL DE LAS ESCUELAS DE SALUD PÚBLICA EN LA EDUCACIÓN MÉDICA*}

\section{RESPONSABILIDAD EN LA FORMACIÓN DE MÉDICOS}

Las escuelas de salud pública tradicionalmente se mantuvieron al margen de las facultades de medicina en lo que atañe a la formación de médicos. Como resultado de un proceso complejo, las facultades de medicina resistían la incorporación de la salud pública, tanto desde el punto de vista conceptual cono institucional, en tanto que las escuelas de salud pública reaccionaban aislándose cada vez más.

En los últimos años, como consecuencia de considerarlas como elementos de un mismo sistema, se registra una creciente integración entre ambas que parece ser una tendencia de gran vigencia futura.

La relación de las escuelas de salud pública con las facultades de medicina, si bien asume características decisivas cuando su dependencia es universitaria, debe existir igualmente, sea cual fuere el ámbito administrativo a que pertenecen. Una escuela de salud pública, por el hecho de depender de un ministerio de salud pública, no pierde responsabilidad en lo referente a la educación médica de pregrado.

La relación de las escuelas de salud pública con las facultades de medicina, por otra parte, debe visualizarse en el amplio contexto de las transformaciones que está sufriendo la educación médica así como la salud pública y su enseñanza.

En pocas palabras se podría decir que los cambios ocurridos en los últimos años en el campo de la educación médica están dirigidos a formar

\footnotetext{
*La sección reimpresiones en esta ocasión publica un artículo escrito por los directores de las Escuelas de Salud Pública de la Universidad de Buenos Aires y la Universidad de Chile, sobre el rol de las escuelas en la formación médica, presentado en 1969 y publicado en la Revista Educación Médica y Salud.

Nos impresiona este trabajo cooperativo trasandino entre dos figuras directivas, apremiadas por el trabajo y las vicisitudes de su tiempo. Ojalá esta publicación sirva para animarnos a comprender la cordillera y el continente como camino o red, y no como muro.

Nos animó su comprensión de la incorporación de las ciencias sociales en los planes de estudio, que "no puede ser sino un primer paso que debe ser sustituido rápidamente por una enseñanza verdaderamente integrada”. Muchas veces nos felicitamos de estar avanzando en la formación de esos ramos en los primeros años de las carreras de la salud y los autores convocados a sus syllabus. Pero como dicen Sonis y Behm la tarea allí recién comienza.

Finalmente, queremos remarcar la claridad con que ellos plantean el estilo de docentes deseado, poniendo en primer lugar imaginación, luego conocimiento y finalmente, dedicación:
} 
un tipo de profesional diferente del que se ha venido formando. Dentro de este proceso, es posible que lo más importante sea orientar la enseñanza en función de las características del médico que se pretende formar.

Hasta hace poco, las facultades de medicina no definían explícitamente el tipo de médico que querían obtener; el egresado salía preparado para atender la patología del individuo enfermo, en servicios hospitalarios o en clínicas privadas, según sus recursos, como si este individuo fuera un ente aislado de la sociedad donde vive, y como si la sintomatología que lo aquejaba fuese el centro de gravedad de la salud. El tipo de médico que se tiende a formar hoy día es, por el contrario, el de un médico líder de un equipo de salud, con conocimientos de la patología y de la realidad sanitaria de la comunidad en que vive y en la cual debe actuar, apto para el manejo de los recursos comunitarios que contribuyen al mantenimiento y a la recuperación de la salud, con un concepto integral de ésta, con una conciencia clara de su misión como universitario, y con capacidad para administrar y adiestrar al personal que trabaja a sus órdenes.

En la formación de este tipo de médico, las escuelas de salud pública, si bien no tienen la responsabilidad primordial, deben desempeñar un rol importante por ser, en muchos casos, los centros donde mayor desarrollo han adquirido los conceptos y las técnicas que sirven de base a la nueva orientación. Como ejemplo de tal aseveración podrían mencionarse las siguientes áreas:
1. El enfoque del fenómeno salud-enfermedad con criterio científico requiere que se tengan suficientes conocimientos de estadística y epidemiología. Para el enfoque de la salud y la enfermedad como epifenómenos del ambiente en el cual el individuo se mueve, es indispensable el conocimiento epidemiológico, cuya mejor puerta de entrada en la educación médica son las escuelas de salud pública. No se nos escapa tampoco la importancia que las ciencias sociales tienen en este asunto, dada la utilidad que para el profesional tiene el conocer la sociedad y el hombre que atiende, y entender y participar eficientemente en las organizaciones sociales que son los servicios de salud.

2. Para el nuevo tipo de médico es fundamental conocer el sistema de atención médica en el cual deberá actuar, así como tener una idea clara de las instituciones que operan en el campo de la salud y de su funcionamiento, a fin de aprovechar todos los recursos comunitarios para la atención de la salud. Esto evitará que solo unos cuantos médicos se dediquen a la administración de servicios y que la gran mayoría de ellos se mantenga totalmente ajena al manejo del sistema de atención del cual forman parte y del que constituyen los niveles operativos.

3. Hace ya algún tiempo se viene sosteniendo que uno de los problemas más serios de la medicina actual y que tiene su base en la educación médica, radica en la fragmentación de los conocimientos como consecuencia de su creciente complejidad.

\footnotetext{
“Quizá debamos recordar, a riesgo de parecer insistentes, que la formación de los docentes aptos para poner en marcha experiencias de este tipo, con la imaginación, el conocimiento y la dedicación que las mismas requieren, constituye el punto clave en el éxito de los cambios buscados".

Sea esta reimpresión un homenaje a estos dos maestros y un guiño a la búsqueda de la imaginación sociológica, que siempre nos convoca. En la deriva del proceso editorial, nos contactamos con Abraam Sonis en la Universidad Maimónides, quien nos señaló: "Hugo Behm fue uno de los mas brillantes maestros de una generacion de sanitaristas chilenos, pioneros de la Salud Pública en América Latina. Todos aprendimos a su lado- de sus conocimientos, de su calidad personal, de su generosidad- y recibimos el estímulo de su amistad y su enseñanza.

"Por aquellos años me sentí muy orgulloso que la OPS nos pidiera que escribiéramos juntos ese documento (¡hace 44 años!), para mí fue como alcanzar la mayoría de edad en la Salud Pública y hoy me conmueve el recuerdo”.

Un agradecimiento explícito a José Fernández, quien siempre nos sorprende con maravillas bibliográficas y que nos mostró este artículo. Yuri Carvajal.
} 
4. La tendencia hacia la especialización, que ha permitido profundizar los conocimientos en la medicina y que ha hecho posible una mejor atención médica, lleva en sí un riesgo que repetidamente se ha hecho notar y que es la fragmentación en la visión del hombre y del enfermo, así como el aislamiento del médico dentro de los límites de su especialidad.

5. Tal tendencia, que sin duda ha de continuar en el futuro y que quizá sea necesario que continúe, debe ser contrabalanceada por enfoques de síntesis que consideren al hombre y a su ambiente como un todo. Probablemente el sanitario sea uno de estos enfoques de síntesis que permite visiones conjugadas de la realidad que se consideran indispensables para evitar la fragmentación señalada. La comprensión de la importancia de la interacción hombre-sociedad podrá poner a cubierto al médico de las visiones unilaterales, tan comunes hoy; quizá pueda evitarse así que el estudiante y, más adelante, el médico se ocupe sólo del órgano enfermo. Este enfoque debe adquirirse desde el comienzo de la carrera.

6. La demanda creciente de servicios médicos por parte de núcleos cada vez más amplios de población; el aumento de la expectativa de vida sin agregar vitalidad a los años adicionales que se viven; el incremento de las afecciones crónicas y degenerativas, y el aumento del costo de la atención médica, son algunos de los muchos factores que han obligado a dar énfasis a la medicina preventiva.

El éxito obtenido en la prevención de las enfermedades transmisibles da base para proyectar hacia las no transmisibles la idea de una prevención eficiente y a bajo costo. Sin embargo, la tecnología médica moderna no siempre significa una medicina preventiva con un rendimiento de nivel aceptable.

La nutrida experiencia de casi todas las escuelas de medicina revela que no es fácil implementar la enseñanza de los aspectos preventivos; en la mayoría de los casos su enseñanza como materia separada de la clínica la transforma en un barniz que el alumno no internaliza y que nunca aplica en su práctica posterior. Su enseñanza durante toda la carre$\mathrm{ra}$, marca un etapa intermedia que creemos se está cumpliendo en la actualidad, y que deberá ser seguida por una integración de la enseñanza de aspectos preventivos y colectivos con los curativos e individuales de la medicina.

La participación de las escuelas de salud pública en esta integración puede resultar muy importante dado que, en gran medida, la misma debe apoyarse en un enfoque clínico-epidemiológico de la afección en cada paciente. Creemos que este enfoque clínicoepidemiológico señala una orientación básica para la medicina del futuro a través de investigaciones que abren la posibilidad a enfoque más integrales de la patología así como de su prevención. Por otra parte, la aplicación de estos enfoques preventivos a la totalidad o a la gran mayoría de la población, que es el campo de actividad de la salud pública, también debe ser presentada en forma integrada a los estudiantes de medicina en las unidades hospitalarias.

Además de las consideraciones anteriores, debe tomarse en cuenta que en el continuum que es la formación y perfeccionamiento de los cuadros profesionales de salud, la vinculación de los distintos grupos facilita un programa integral de largo alcance, fortalece la formación de grupos académicos funcionales y estimula el perfeccionamiento de la enseñanza y de los mismos profesores.

En la consecución de los fines señalados, sería recomendable que las escuelas de salud pública realizaran, entre otras, las siguientes actividades:

1. Dictar cursos o unidades de enseñanza destinados a la formación y perfeccionamiento de los docentes de las escuelas de medicina, en especial de los profesores de medicina preventiva, medicina social u otras disciplinas similares.

2. Participar en el proceso de revisión y perfeccionamiento de los programas de formación de médicos y otros profesionales del sector salud, de modo que ellos lleven a la producción de profesionales adecuados en cantidad y calidad a las necesidades del país. 
3. Fomentar y participar en investigaciones que contribuyan a:

a) definir las necesidades de profesionales y otros grupos técnicos en el sector salud; b) precisar los objetivos de la educación de estos profesionales, determinando las características que deben tener los egresados de acuerdo con las condiciones actuales y futuras (deseables) de la atención médica en el país; y c) evaluar el logro de estos objetivos e impulsar el continuo perfeccionamiento de los planes educacionales pertinentes.

4. De un modo más general, participar en la definición de una política de formación y perfeccionamiento de los recursos humanos en el sector salud, y determinar las responsabilidades que las distintas instituciones deben asumir en ella.

No cabe duda que estas modificaciones de la educación médica repercutirán en la docencia de postgrado de la salud pública que hasta ahora ha debido, en gran medida, suplir las deficiencias formativas previas.

La experiencia de las distintas escuelas de medicina revela que los alumnos pueden adquirir, a nivel de pregrado, conceptos a los cuales el curso de salud pública debería dedicar parte de su tiempo. Una vez que los médicos se hayan formado bajo planes de estudio actualizados, la enseñanza de postgrado de la salud pública podrá y deberá orientarse hacia la profundización de técnicas que hoy no es posible encarar por falta de tiempo.

\section{RELACIONES ESTRUCTURALES CON LAS FACULTADES DE MEDICINA Y LA UNIVERSIDAD}

No resulta fácil discernir sobre fórmulas operativas eficaces en relación con este tema, dado que los aspectos conceptuales y administrativos se influyen mutuamente. En gran medida, la organización futura de las escuelas deberá acomodarse a las modificaciones que surjan de los cambios en la enseñanza, y el éxito que se alcance con ta- les cambios dependerá de lo adecuado que resulten las estructuras. Además, el distinto panorama que presenta cada una de las facultades y universidades no permite fijar directivas o fórmulas de aplicación común. Se estima conveniente, por lo tanto, señalar algunos conceptos generales.

La departamentalización, que ha tomado tanto auge en los últimos años, tiene un valor relativo si la juzgamos aisladamente. Pierde sentido si no resulta, por una parte, de una redefinición de los fines y funciones de la universidad ante la sociedad y, por la otra, del consiguiente cambio de actitudes y de la plena conciencia que de su rol tienen los integrantes de la universidad. Requiere, asimismo, una conducción de la universidad en la cual tengan participación responsable todos los componentes de la comunidad universitaria de acuerdo con la idiosincrasia de cada uno de ellos.

En líneas generales, las experiencias que se decida poner en marcha deberán coincidir con la realidad de cada caso y estar de acuerdo con el tamaño, la estructura, la organización y las características político-culturales de cada organismo universitario en particular. No presenta el mismo panorama una universidad de reciente creación que otra de larga tradición y estructura cristalizada, ni tampoco una institución que agrupa miles de alumnos que otra pequeña. Lo que es inevitable es la puesta en marcha de nuevas experiencias en todas y cada una de ellas.

Quizá éste sea uno de los casos en los cuales la apreciación de la coyuntura adquiere un valor fundamental. Las características y el estado por el cual pasa cada una de las instituciones, adquieren una importancia fundamental en la puesta en marcha de los cambios. Daría la impresión de que en las universidades con gran número de alumnos, ubicadas en grandes ciudades, la departamentalización de toda la universidad presentaría inconvenientes casi insuperables, y la vía operativa parecería ser la departamentalización de las facultades.

La existencia de problemas administrativos, políticos o estratégicos, obliga, en muchos casos, a introducir los cambios de un 
modo paulatino. La incorporación de determinadas disciplinas (epidemiología, demografía, etc.) en forma de asignaturas, puede transformarse en un factor contrario al cambio estructural si lleva implícita la creación de una nueva cátedra, ya que vendría a reforzar así la estructura tradicional de las instituciones.

La falta de ortodoxia en lo que se refiere a una modificación óptima del plan de estudios, así como la necesidad de proceder a la promoción de cambios sin alteraciones drásticas y mientras la enseñanza prosigue, obliga a ensayar esquemas prácticamente originales en cada uno de los casos. Esto tiene especial aplicación si lo referimos a la ubicación de una escuela de salud pública como organismo docente de posgrado que al mismo tiempo tiene responsabilidad en la docencia de pregrado en una escuela de medicina.

En efecto, en los últimos tiempos ha habido una tendencia a unificar las escuelas de salud pública con las cátedras de higiene y medicina preventiva en departamentos responsables de la enseñanza, tanto en el pre como en el postgrado. Si bien las experiencias son recientes, esta combinación parece señalar la vía operativa más indicada para el futuro, dado lo promisorio de los resultados que se comienzan a apreciar en las facultades que las han puesto en práctica. Se comienza a romper la barrera entre la enseñanza de pre y postgrado, se intercambian experiencias en un equipo multiprofesional y multidisciplinario, hay flexibilidad y permeabilidad para el mejor uso de recursos comunes, y se definen políticas de docencia, investigación y formación académica en un contexto más amplio y más estimulante.

Quizá, para las escuelas de salud pública, ello signifique pérdida de independencia y de agilidad administrativa en el manejo de las actividades de postgrado, pero, aparte de que pueden tomarse precauciones que permitan un manejo eficiente del departamento, estimamos que el precio vale la pena, comparado con los beneficios que se pueden obtener.

Para aquellos casos en que la solución arriba descrita aún no es posible, restan todos los mecanismos de coordinación. Lo impor- tante es que las escuelas de salud pública sientan la necesidad e impulsen estas relaciones de trabajo sin mantenerse enquistadas en lo que fueron sus funciones tradicionales.

Un punto crítico en este campo lo constituye la necesidad de formar personal docente capaz de llevar a cabo las tareas señaladas. La enseñanza de la epidemiología para una visión práctica de la medicina preventiva, la multicausalidad en el estudio de la patología, la enseñanza del fenómeno salud-enfermedad en relación con todas las variables del medio, etcétera, exigen una interrelación estrecha entre materias básicas y clínicas así como con disciplinas que forman la salud pública. Quizá el trabajo más importante en todo este proceso sea la formación de personal de alto nivel necesario para encarar con éxito este cambio, y ello deberá realizarse a través del trabajo conjunto de los docentes de todas las disciplinas, cualquiera sea su campo. En el hospital docente del futuro, que será asiento de todas las disciplinas que confluyen en una escuela de medicina, incluso las básicas, tendrá su lugar el especialista en las distintas orientaciones de la salud pública (administración, estadística, epidemiología), integrado al resto del equipo de salud, no sólo en la docencia sino también en trabajos de investigación y en los programas de terreno.

El problema, en la práctica, concierne, pues, a los mecanismos que puedan establecer mejores relaciones funcionales entre los docentes de la escuela de salud pública y las escuelas de medicina, haya o no integración. Entres estos procedimientos adquiere importancia la discusión conjunta de los fines y funciones de la universidad en general, y en particular de las facultades y escuelas del sector salud frente a la sociedad. En el mismo sentido, cabe señalar la elaboración conjunta de los planes de enseñanza que, en cuanto definen objetivos precisos derivados del estudio de la situación nacional, permiten visualizar el fin perseguido más allá de la barrera de la especialización.

La formación y el perfeccionamiento de los docentes con esta visión global, de conjunto, encuentra sus posibilidades de realización a través del propio desarrollo de un programa de enseñanza médica integrador. 
Los docentes clínicos y de salud pública que acompañan a los alumnos en una práctica en equipo que se extienda del hospital docente a la comunidad o a hospitales rurales, están aprendiendo, tanto como ellos, a trabajar en equipo para una medicina integral.

\section{RELACIONES CON OTRAS ESCUELAS Y PROFESIONES}

Estas relaciones se dan en un marco de gran amplitud y de distintos niveles y, aun para el más general de los enfoques, debemos hacer una primera distinción entre aquellas especialmente conexas por compartir una base biológica común, como medicina, odontología, enfermería, farmacia, etc., y aquellas otras con las que encontramos áreas de convergencia, tales como las ciencias sociales, políticas, económicas o exactas.

Por supuesto que la ya citada departamentalización de facultades y universidades, constituye la base y fija el marco dentro del cual se deben considerar estas relaciones. Asimismo, y más allá del problema de las estructuras y de la organización, lo que debiera unir a los universitarios no son solamente los aspectos comunes de sus respectivas disciplinas, sino la internalización de los fines y las funciones generales de la universidad, pudiendo ser este uno de los productos más interesantes del proceso de cambio que están experimentando las universidades.

\section{Relación con disciplinas conexas}

Desde el punto de vista de la salud, el problema debe enfocarse teniendo como directiva central la formación del equipo. Por largo años en salud pública se ha insistido en la necesidad de ejecutar las actividades a través del equipo de salud, y con toda razón se insiste también en que no debe hablarse del médico sino del equipo que se necesita.

La experiencia demuestra que el intento de formar equipos con personal adiestrado de acuerdo con los criterios tradicionales hoy en vigencia, resulta sumamente difícil dado el encasillamiento y la deformación profesional con que egresan los alumnos de las distintas carreras.

El estudio de las distintas instituciones que se desenvuelven en nuestro campo, revela claramente en qué medida la gran mayoría de los problemas que surgen y que dificultan el cumplimiento de las actividades, se deben a una patología institucional cuyo origen reside en la deformación profesional de sus componentes, difícil de corregir una vez producida, dada su rigidez. Las modificaciones que se buscan favorecerán, por el contrario, la adaptación al trabajo en equipo. No cabe duda que un médico que egrese identificado con los objetivos que hoy se pretenden para la educación médica, buscará y aprovechará todas las posibilidades de trabajar en equipo, y lo mismo puede decirse de las otras profesiones.

Por supuesto que esta formación de médicos, enfermeras odontólogos, etcétera, naturalmente aptos para el trabajo en equipo, significa modificaciones fundamentales en la enseñanza. Una vez más, formación de recurso humanos y manejo de los servicios aparecen como un sistema que debe ser visto integralmente. Tal como se especifica más arriba, las modificaciones que deben realizarse en los hospitales para transformarlos en centros de salud comunitarios, transforman de por sí el ámbito de trabajo de los estudiantes, llevándonos a un tipo de enseñanza en la cual la integración en equipo, además de producirse naturalmente, es condición básica para su realización.

\section{Relación con disciplinas convergentes}

Huelga insistir en la importancia que las ciencias sociales, económicas, políticas, etcétera, tienen en las actividades de salud y, en consecuencia, en la enseñanza de las profesiones responsables del cuidado de la misma.

La experiencia de los últimos años muestra no sólo la prevalencia de los factores económicos, sociales o políticos en el nivel de salud de una región, sino también, desde un punto de vista muy concreto, su importancia en la ejecución de los 
programas del sector salud. Muchos de ellos han fracasado por no tener en cuenta -y esto se ha dicho hasta el cansancio- las condiciones que influyen sobre el marco en el cual se desenvuelven las actividades de salud, indispensables para una visión global de los problemas. Lo que importa señalar es que estas condiciones no deben ser vistas como exteriores al sistema salud, sino como constituyentes de la realidad con la cual debemos trabajar.

Como factor promisorio cabe señalar el gran interés que los especializados en esta materia empiezan a mostrar por el sector salud y el número creciente de trabajos interdisciplinarios que comienzan a aparecer. Sin embargo, estos profesionales han sido educados en el criterio parcial que caracteriza la formación profesional actual, y su integración al campo de la salud requiere adaptación previa. Múltiples procedimientos se están poniendo en práctica a fin de concretar esta adaptación: desde seminarios y cursillos, hasta la realización del curso básico de salud pública, pasando por trabajos de investigación en común. Esto está siendo complementado por estudios formales que numerosos médicos especializados en salud pública realizan en estas disciplinas a fin de concretar las áreas comunes.

La necesidad de introducir las ciencias sociales en la enseñanza de la medicina, ha llevado en ocasiones a su incorporación en forma de asignaturas en los planes de estudio, lo cual si bien significa un sensible adelanto, no puede ser sino un primer paso que debe ser sustituido rápidamente por una enseñanza verdaderamente integrada, objetivo que se espera lograr en los próximos años. Las experiencias en marcha son realmente reveladoras de interesantes posibilidades al respecto; solo nos permitimos subrayar que este trabajo de integración de los planes de estudio debe basarse en experiencias concretas y prácticas llevadas a cabo en áreas particulares $y$, reconocida la falta de ortodoxia, debe ser objeto de revisiones periódicas, a medida que se vayan observando los resul- tados. Quizá debamos recordar, a riesgo de parecer insistentes, que la formación de los docentes aptos para poner en marcha experiencias de este tipo, con la imaginación, el conocimiento y la dedicación que las mismas requieren, constituye el punto clave en el éxito de los cambios buscados.

\section{ESCUELAS DE SALUD PÚBLICA, UNIVERSIDAD Y DESARROLLO}

El rol de las escuelas de salud pública en relación con el desarrollo, debe ser considerado dentro del cuadro general de las relaciones de la universidad con la sociedad y sus problemas. La escuela de salud pública, al formar parte de una universidad, incrementará las posibilidades de cumplir con su responsabilidad y, al mismo tiempo, contribuirá a que el organismo universitario se incorpore a la solución de los problemas de la comunidad a la cual pertenece. En el marco que señala esta doble relación, nos permitimos incluir algunos matices que juzgamos interesantes:

1. Han sido materia de discusión las posibilidades reales del sector salud para mejorar el nivel de salud de una población, dado el valor que tienen en la determinación del mismo los factores socioeconómicos condicionantes.

Por un lado, existe una corriente de opinión que insiste en la importancia que tiene una programación adecuada, una óptima distribución de recursos y una ejecución eficiente de las actividades, en el mejoramiento del nivel de salud de una población.

Otra corriente de opinión, por el contrario, afirma que son los factores condicionantes los fundamentales, y que lo que pueda conseguirse exclusivamente del sector salud no es significativo, sobre todo en aquellos países o áreas con niveles de ingresos muy bajos.

En el momento de asumir una posición, el especialista en salud pública no puede ignorar los conocimientos y las técnicas a su disposición, los cuales debe 
utilizar al máximo, tratando de obtener todo el provecho posible de los recursos a su cargo; pero también debe estar consciente de las limitaciones que existen para solucionar los problemas desde el sector salud. Ante este planteo, hay consenso en que el mejoramiento del nivel de vida, consecuencia de la promoción económico-social, es indispensable para asegurar mejores condiciones de salud.

Las escuelas de salud pública, por lo tanto, deberán contribuir a este proceso, formando profesionales que, a la vez que posean un alto nivel técnico, contribuyan a transformar las universidades en un factor promotor del desarrollo.

2. Como el desarrollo económico-social es fundamentalmente un cambio de estructuras, se ha dicho, y con razón, que todo el proceso puede considerarse como un conflicto entre los partidarios de una sociedad rígida y cerrada y los que abogan por una sociedad abierta y de gran movilidad que posibilite los cambios. Este conflicto se plantea en todos los sectores y la opción, sea explícita o no, es inevitable.

Para quienes se desempeñan en el campo de la salud, optar por una sociedad abierta significa contribuir a solucionar los problemas de salud al favorecer las modificaciones que llevan al mejoramiento del nivel de vida de la población. La coherencia consigo mismo exige, pues, del sector salud, una alianza natural con quienes en sus respectivos sectores comparten lo que se ha dado en llamar la "coalición modernizante".

3. Si la universidad ha de jugar un papel de primera línea en el desarrollo de un país, cada una de sus escuelas debe cumplir funciones significativas en el área de su incumbencia, ya sea asesorando o investigando aquellos aspectos que son esenciales para el éxito de todo el proceso.

En el caso particular de la salud pública, los programas pueden adquirir gran valor si éstos significan el desenca- denamiento de un proceso de desarrollo comunitario. Programas de salud que persigan como único objetivo la reducción parcial de los indicadores que señalan las malas condiciones de vida y de salud de una región, dejando a un lado la solución de condiciones responsables de estos indicadores y de los estrangulamientos o rigideces que impiden un cambio en las estructuras, son programas destinados al fracaso aunque obtengan éxitos temporarios. Por tanto, la importancia de los programas de salud, deberá ser medida, no sólo por los resultados cualitativos que introducen o producen en la comunidad, los cuales promueven tanto su organización como su desarrollo.

4. Las acciones de la universidad tienen grandes repercusiones en la comunidad nacional por la jerarquía intelectual de quienes forman parte de ella, en virtud de lo cual, el efecto de demostración que pueden alcanzar tomas de posición, actitudes y formas de trabajo que la universidad desarrolle, adquieren gran trascendencia dentro de la sociedad. El hacer suyos los objetivos del desarrollo económico-social, el tratar de alcanzarlos por la suma de esfuerzos de numerosas disciplinas sin que interfieran diferencias políticas o ideológicas, puede servir de ejemplo claro de cómo pueden unirse los esfuerzos en pro del objetivo común.

Las ciencias de la salud, por ser un campo especial de confluencia de disciplinas que se relacionan tanto con el conocimiento biomédico como con el social -en el cual diversos profesionales trabajan organizadamente al servicio del bienestar de la comunidad y de cada uno de los individuos que la componen- deben formar a sus alumnos teniendo en cuenta esta directivas, haciendo posible en esta forma que sus egresados se conviertan en verdaderos líderes de los cambios que se propician en la comunidad en la cual actúan. 